\title{
Optical method to detect the relationship between chirality of reciprocal space chiral multifold fermions and real space chiral crystals
}

\author{
Yan Sun $\odot,{ }^{1, *}$ Qiunan $\mathrm{Xu},{ }^{1}$ Yang Zhang $\odot,{ }^{2}$ Congcong Le, ${ }^{1}$ and Claudia Felser $\circledast^{1,3}$ \\ ${ }^{1}$ Max Planck Institute for Chemical Physics of Solids, 01187 Dresden, Germany \\ ${ }^{2}$ Department of Physics, Massachusetts Institute of Technology, Cambridge, Massachusetts 02139, USA \\ ${ }^{3}$ Center for Nanoscale Systems, Faculty of Arts and Sciences, Harvard University, 11 Oxford Street, LISE 308 Cambridge, \\ Massachusetts 02138, USA
}

(Received 6 January 2020; accepted 25 August 2020; published 23 September 2020)

\begin{abstract}
The chirality of chiral multifold fermions in reciprocal space is related to the chirality of crystal lattice structures in real space. In this study, we propose a strategy to detect and identify multifold fermions of opposite chirality in nonmagnetic systems using second-order optical transports. Chiral crystals related with inversion operations cannot be made to overlap with each other via any experimental operation. Further, chiral multifold fermions within such crystals host opposite chiralities corresponding to a given $k$ point. A change in chirality is indicated by a corresponding change in the sign of the second-order charge current dominated by chiral fermions. This property can be exploited to study the relationship between chiralities in reciprocal and real spaces by utilizing bulk transport.
\end{abstract}

DOI: 10.1103/PhysRevB.102.104111

\section{BACKGROUND AND INTRODUCTION}

Multifold massless fermions with nonzero topological charge have garnered significant attention in the field of topological materials. In contrast with fourfold degenerate Dirac fermions and doubly degenerate Weyl fermions, which have directly analogous fundamental particles in high-energy physics, multifold fermions do not follow the Poincaré symmetry. Instead, they follow crystal symmetry; hence, they do not have counterparts among real particles [1,2]. More interestingly, multifold fermions in chiral crystals can host nonzero topological charges with Chern numbers greater than 1 , which induces both nontrivial topological surface states and exotic bulk transport properties [2].

Since multifold fermions are located at high-symmetry points, they guarantee long surface Fermi arcs spanning the entire Brillouin zone (BZ) [3-6]. In addition to the wide separation between opposite topological charges in the momentum space, the absence of mirror symmetry also leads to their large separation in energy space, providing an ideal platform for the study of quantized circular photogalvanic effects (CPGE) $[3,5,7,8]$. Following their theoretical predictions, long Fermi arcs, high-order degenerated band crossings, and topological CPGE were soon observed in the expected chiral crystals via angle-resolved photoemission spectroscopty (ARPES) [9-13], scanning tunneling microscopy (STM) [14], and optical measurements [15-17].

*ysun@cpfs.mpg.de

Published by the American Physical Society under the terms of the Creative Commons Attribution 4.0 International license. Further distribution of this work must maintain attribution to the author(s) and the published article's title, journal citation, and DOI. Open access publication funded by the Max Planck Society.
Recently, based on ARPES and STM measurements, a sign change was detected in the Fermi velocity of surface Fermi arcs in semimetals possessing chiral multifold fermions and crystals of opposite chiralities. This implied a deep relationship between chiral lattices in the real space and chiral fermions in the momentum space $[13,14,18]$. This relationship offers a degree of freedom to modulate chiral fermions and their corresponding physical properties. Hence, it is important to understand the relationship between chiralities in real and reciprocal spaces as well as any associated phenomena. In the present study, based on symmetry analyses and numerical calculations, we provide a strategy to detect the interactions between chiral fermions and chiral crystals from the perspective of transport measurement. Since crystal structures of the same compound with opposite chiralities are related by an inversion operation, the relationship between chiral fermions and chiral crystals can be detected by means of nonlinear optical and electrical transports. Indeed, the extra magnetic field has also been used to tune the linear and nonlinear optical response in magnetic systems, especially the sign change [19-23]. Here we mainly focus on nonmagnetic systems.

To date, all experimentally verified materials with chiral multifold fermions have been observed to belong to the space group $P 2{ }_{1} 3$. For a crystal in the space group $P 2{ }_{1} 3$, based on the twofold screw rotation symmetries $\left(s_{2 x}=\left\{c_{2 x} \mid\left(\frac{1}{2} \frac{1}{2} 0\right)\right\}\right.$, $s_{2 y}=\left\{c_{2 y} \mid\left(0 \frac{1}{2} \frac{1}{2}\right)\right\}$ and $\left.s_{2 z}=\left\{c_{2 z} \mid\left(\frac{1}{2} 0 \frac{1}{2}\right)\right\}\right)$, the corresponding glide mirror operations can be obtained via a simple inversion. Therefore, chiral crystals in the space group $P 2{ }_{1} 3$ with opposite chiralities are related via a simple inversion operation, as depicted in Fig. 1(a) and 1(b). In any other crystal structure without rotation $c_{2}$ or screw $s_{2}$ symmetry, a mirror operation can serve as the equivalent of an inversion operation in combination with an experimental $c_{2}$ rotation or $s_{2}$ screw operation as samples can be rotated by any angle in experiments. 
(a)

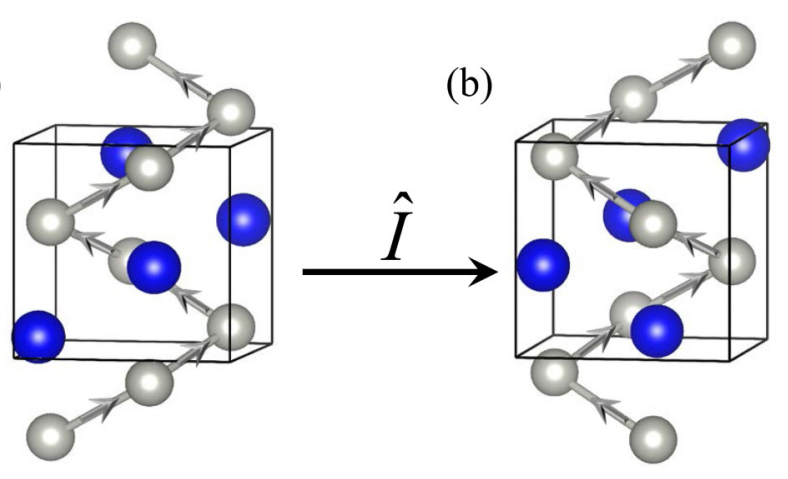

(c)

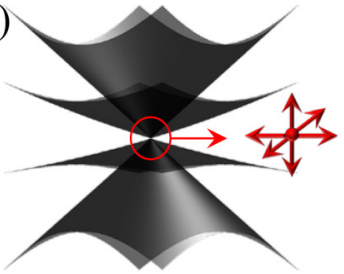

(e)

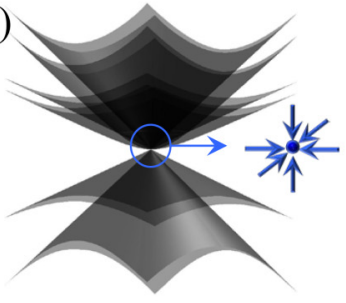

(d)

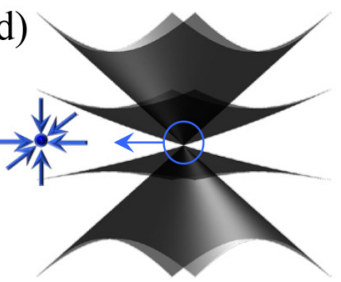

(f)

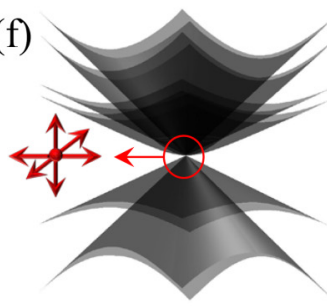

FIG. 1. Inversion of chirality in a chiral crystal structure leads to the inversion of chirality in associated chiral multifold fermions. (a), (b) Crystal lattice structures of semimetals with opposite chiralities in the space group $P 2{ }_{1} 3$ with chiral multifold fermions, considering $\mathrm{RhSi}$ as an example. (c), (d) Schematics of fourfold degenerate fermions with spin-3/2 excitation at the $\Gamma$ point. (e), (f) Schematics of Double threefold degenerate fermions with spin-1 excitation at the $R$ point. The local effective Hamiltonians around the crossing points can be expressed by $H=-\boldsymbol{k} \cdot \boldsymbol{S}_{4 \times 4}, H=\boldsymbol{k} \cdot \boldsymbol{S}_{4 \times 4}, H=-\boldsymbol{k} \cdot \boldsymbol{S}_{3 \times 3}$, and $H=\boldsymbol{k} \cdot \boldsymbol{S}_{3 \times 3}$ for (c)-(f), respectively. We use $\hbar v_{F}=1$ and $\hbar v_{F}=2$ for $H_{3-\text { fold }}^{1}$ and $H_{3 \text {-fold }}^{2}$, respectivley. The parameter $\phi=\pi / 2$ in $H_{3-\text { fold }}$. We use $\hbar v_{F}=1$ and $x=\arctan (-3)$ for $H_{4-\text { fold }}$ Further details of the effective model can be found in Ref. [2,7].

In compounds belonging to the space group $P 2_{1} 3$, the chiral multifold fermions located at $\Gamma$ and $R$ exhibit fourand sixfold degeneracies, respectively [1-6]. Similar to Weyl fermions, these two types of chiral multifold fermions can be described in a unified form using the Hamiltonian $H=$ $\chi \boldsymbol{k} \cdot \boldsymbol{S}$, with $\chi= \pm 1$ [2] and the following pseudospin matrix: $\left[S_{i}, S_{j}\right]=i \epsilon_{i j k} S_{k}$. The fourfold degeneracy at the point, $\Gamma$, is a spin-3/2 excitation with a $4 \times 4$ pseudospin matrix

$$
\begin{aligned}
& H_{4-\text { fold }} \\
& \quad=\left(\begin{array}{cccc}
a k_{z} & 0 & -\frac{a+3 b}{4} k_{+} & -\frac{\sqrt{3}(a-b)}{4} k_{-} \\
0 & b k_{z} & \frac{\sqrt{3}(a-b)}{4} k_{-} & -\frac{a+3 b}{4} k_{+} \\
-\frac{a+3 b}{4} k_{-} & \frac{\sqrt{3}(a-b)}{4} k_{+} & -a k_{z} & 0 \\
-\frac{\sqrt{3}(a-b)}{4} k_{+} & -\frac{a+3 b}{4} k_{-} & 0 & -b k_{z}
\end{array}\right)
\end{aligned}
$$

with $a=\hbar v_{F} \cos x, b=\hbar v_{F} \sin x$, and $k_{ \pm}=k_{x} \pm i k_{y}$, and the topological charge has a Chern number of \pm 4 when the two lower bands are occupied. The sixfold degeneracy at the $R$ can be constructed by double spin-1 Weyl fermions and a doubly degenerate quadratic bands. Hence, a $3 \times 3$ pseudospin matrix

$$
H_{3-\text { fold }}=\hbar v_{F}\left(\begin{array}{ccc}
0 & e^{i \phi} k_{x} & e^{-i \phi} k_{y} \\
e^{-i \phi} k_{x} & 0 & e^{i \phi} k_{z} \\
e^{i \phi} k_{y} & e^{-i \phi} k_{z} & 0
\end{array}\right)
$$

can be used to describe this sixfold degeneracy in the form of

$$
H_{6 \text {-fold }}=\left(\begin{array}{cc}
H_{3 \text {-fold }}^{1} & 0 \\
0 & H_{3-\text { fold }}^{2}
\end{array}\right)
$$

as depicted in Fig. 1(e). Since spin-1 Weyl fermions have a Chern number of \pm 2 , the corresponding topological charge for double spin- 1 Weyl fermions is \pm 4 , inducing the whole system to follow a "no-go theorem." For both spin-1 and spin- $3 / 2$ excitations, the sign of the topological charge is dependent on the sign of the prefactor, $\chi$. Under an inversion operation, $H(\boldsymbol{k})$ is converted to $H(-\boldsymbol{k})$. While retaining the form of energy dispersion, chirality changes the sign, as depicted schematically in Figs. 1(c)-1(f).

Though the chirality of a multifold fermion is reversed by the inversion operation, time-reversal symmetry induces a net zero Berry flux in the entire BZ. Therefore, the anomalous Hall effect cannot be used to investigate the change in chirality. However, the second-order responses are odd with respect to inversion, which suggests a possible method to detect the sign change in chirality.

\section{NONLINEAR HALL EFFECTS}

There are mainly two types of transports based on secondorder responses: nonlinear optical effects and nonlinear Hall effects caused by Berry curvature dipoles [24-28]. The specific crystal symmetry of $P 2{ }_{1} 3$ implies that the off-diagonal elements of the nonlinear Hall conductivity tensor are zero and that there is only one independent diagonal element. However, owing to time-reversal symmetry,

$$
\begin{aligned}
& \int f_{0}\left(D_{x x}+D_{y y}+D_{z z}\right) d k_{x} d k_{y} d k_{z} \\
& =\int f_{0}\left(\frac{\partial \Omega_{x}}{\partial k_{x}}+\frac{\partial \Omega_{y}}{\partial k_{y}}+\frac{\partial \Omega_{z}}{\partial k_{z}}\right) d k_{x} d k_{y} d k_{z} \\
& =\int\left(\Omega_{x} \frac{\partial f_{0}}{\partial k_{x}}+\Omega_{y} \frac{\partial f_{0}}{\partial k_{y}}+\Omega_{z} \frac{\partial f_{0}}{\partial k_{z}}\right) d k_{x} d k_{y} d k_{z} \\
& =\int\left(\boldsymbol{\Omega} \cdot \frac{\partial f_{0}}{\partial \boldsymbol{k}}\right) d k_{x} d k_{y} d k_{z} \\
& =\int \delta\left(E-E_{F}\right) \boldsymbol{\Omega} \cdot d \boldsymbol{k} \\
& =0
\end{aligned}
$$

implies that the trace of Berry curvature dipole is zero. Consequently, nonlinear Hall effects induced by the Berry curvature dipole vanish in materials belonging to the space group $P 2{ }_{1} 3$. Therefore, we consider second-order optical transports, CPGE, linear photogalvanic effects (LPGE), and 
second-harmonic generation (SHG) to investigate responses to the reversal of chirality of multifold fermions.

\section{SYMMETRY AND MICROSCOPIC ANALYSIS}

In crystals without inversion centers, polarized light can generate DC photocurrent and second-harmonic dipoles in the material [24-26,29-37]. In contrast to the photovoltaic effect in $p-n$ junctions, the photovoltaic effect induced by polarized light is dependent solely on the bulk band structure and is not limited by the band gap. Therefore, the polarized-light-induced photoelectric effect is also called the bulk photovoltaic effect. Depending on the polarization of the incident light, the generated DC photocurrent can be classified into two types: an injection current induced by circularly polarized light $\frac{d \boldsymbol{j}_{a}}{d t}=\chi_{b c}^{C, a}(0, \omega,-\omega) E_{b}(\omega) E_{c}(-\omega)$ and a shift current induced by linearly polarized light $\boldsymbol{j}_{a}=$ $\chi_{b c}^{L, a}(0, \omega,-\omega) E_{b}(\omega) E_{c}(-\omega)[26]$, where $E_{i(j, k)}(\omega)$ denotes an electrical field with $i, j, k=x, y, z$.

First, we check the response of the local $k$-space distribution of these two nonlinear optical conductivities to the inversion of chirality. The CPGE tensor is purely imaginary and can be expressed as [26]

$$
\begin{aligned}
\chi_{b c}^{C, a}(0, \omega,-\omega)= & \frac{e^{3} \pi}{\hbar V} \sum_{k} \sum_{m, n} f_{n m}^{k} \Delta_{k, m n}^{a}\left[r_{k, m n}^{c}, r_{k, n m}^{b}\right] \\
& \times \delta\left(\hbar \omega-E_{k, m n}\right),
\end{aligned}
$$

and the LPGE tensor is real and can be expressed as [26]

$$
\begin{aligned}
\chi_{b c}^{L, a}(0, \omega,-\omega)= & \frac{i e^{3} \pi}{\hbar V} \sum_{k} \sum_{m, n} f_{n m}^{\boldsymbol{k}}\left(r_{\boldsymbol{k}, m n}^{b} r_{\boldsymbol{k}, n m}^{c ; a}\right. \\
& \left.+r_{\boldsymbol{k}, m n}^{c} r_{\boldsymbol{k}, n m}^{b ; a}\right) \delta\left(\hbar \omega-E_{\boldsymbol{k}, m n}\right),
\end{aligned}
$$

with

$$
r_{\boldsymbol{k}, n m}^{a}=i\left\langle n\left|\partial_{k a}\right| m\right\rangle=\frac{v_{\boldsymbol{k}, n m}^{a}}{i \omega_{\boldsymbol{k}, n m}}
$$

and

$$
\begin{aligned}
r_{\boldsymbol{k}, n m}^{a ; b}= & \partial_{k b} r_{\boldsymbol{k}, n m}^{a}+\left(\left\langle n\left|\partial_{k b}\right| n\right\rangle-\left\langle m\left|\partial_{k b}\right| m\right\rangle\right) r_{\boldsymbol{k}, n m}^{a} \\
= & \frac{i}{\omega_{\boldsymbol{k}, n m}}\left[v_{\boldsymbol{k}, n m}^{a} \Delta_{\boldsymbol{k}, n m}^{a}+v_{\boldsymbol{k}, n m}^{b} \Delta_{\boldsymbol{k}, n m}^{b}-\omega_{\boldsymbol{k}, n m}^{a b}\right. \\
& \left.+\sum_{p \neq n, m}\left(\frac{v_{\boldsymbol{k}, n p}^{a} v_{\boldsymbol{k}, p m}^{b}}{\omega_{\boldsymbol{k}, p m}}-\frac{v_{\boldsymbol{k}, n p}^{b} v_{\boldsymbol{k}, p m}^{a}}{\omega_{\boldsymbol{k}, n p}}\right)\right],
\end{aligned}
$$

where $f_{k, n m}^{k}=f_{n}^{\boldsymbol{k}}-f_{m}^{\boldsymbol{k}}$ denotes the difference between two bands of the Fermi-Dirac distribution, $E_{k, m n}=E_{k, n}-E_{k, m}$ denotes the energy difference between the bands, $v_{k, n m}^{a}=$ $\frac{1}{\hbar}\left\langle n\left|\partial_{a} \hat{H}\right| m\right\rangle$ denotes the velocity matrix, $\Delta_{k, n m}^{a}=v_{\boldsymbol{k}, n n}^{a}-$ $v_{\boldsymbol{k}, m m}^{a}$ denotes the difference in Fermi velocity between the bands, and $\omega_{\boldsymbol{k}, n m}^{a b}=\frac{1}{\hbar^{2}}\left\langle n(\boldsymbol{k})\left|\partial_{a b}^{2} H\right| m(\boldsymbol{k})\right\rangle$. For the convenience of analysis of the microscopic relationship between the band structure and second-order conductivity, we set

$$
\begin{aligned}
\tilde{\chi}_{b c}^{C, a}(\boldsymbol{k} ; 0, \omega,-\omega)= & \sum_{m, n} f_{n m}^{\boldsymbol{k}} \Delta_{\boldsymbol{k}, m n}^{a}\left[r_{\boldsymbol{k}, m n}^{c}, r_{\boldsymbol{k}, n m}^{b}\right] \\
& \times \delta\left(\hbar \omega-E_{\boldsymbol{k}, m n}\right)
\end{aligned}
$$

and

$$
\begin{aligned}
\tilde{\chi}_{b c}^{L, a}(\boldsymbol{k} ; 0, \omega,-\omega)= & \sum_{m, n} f_{n m}^{\boldsymbol{k}}\left(r_{\boldsymbol{k}, m n}^{b} r_{\boldsymbol{k}, n m}^{c ; a}+r_{\boldsymbol{k}, m n}^{c} r_{\boldsymbol{k}, n m}^{b ; a}\right) \\
& \times \delta\left(\hbar \omega-E_{\boldsymbol{k}, m n}\right) .
\end{aligned}
$$

An inversion operation changes the position $(x, y, z) \rightarrow$ $(-x,-y,-z)$ in real space and $\left(k_{x}, k_{y}, k_{z}\right) \rightarrow\left(-k_{x},-k_{y},-k_{z}\right)$ in reciprocal space. Therefore, $r_{k, n m}^{a}$ is odd with respect to inversion. Similarly, the term $\Delta_{k, n m}^{a}$ is also odd with respect to inversion. Another critical term, $r_{k, n m}^{a ; b}$, changes the sign twice under inversion. As a result, it is even with respect to inversion. Therefore, for a second-order response, $\boldsymbol{j}_{a}=\chi_{b c}^{a} E_{b} E_{c}$, the sign of the conductivity, $\chi$, will become negative upon inversion of each of its coordinate indices. For the specific space group $P 2{ }_{1} 3$, the screw $s_{2}$ symmetry $s_{2 z}$ changes the sign of the optical conductivity tensor, with the $z$ index appearing an even number of times, making them zero. Similarly, when the $x$ or $y$ index appears an even number of times, the elements with that index vanish because of $s_{2 x}$ or $s_{2 y}$, respectively. Further, owing to the $D_{2}$ subgroup, the three indices yield the identical optical conductivity, and only one nonzero independent tensor element of each index remains for both second-order CPGE and LPGE: $\chi_{y z}^{C(L), x}=\chi_{z x}^{C(L), y}=\chi_{x y}^{C(L), z}$. Thus, considering the sixfold degeneracy at the point, $R$, as an example, we analyze the variation in the distribution functions of $\tilde{\chi}_{y z}^{C(L), x}(\boldsymbol{k} ; 0, \omega,-\omega)$ with respect to the inversion operation.

To perform the numerical analysis, we use the reported tight-binding model for RhSi [3]

$$
H(\boldsymbol{k})=H_{0}(\boldsymbol{k})+\sum_{i=1,2,3}\left(V_{r, i}(\boldsymbol{k})+V_{s, i}(\boldsymbol{k})\right)
$$

with

$$
\begin{aligned}
H_{0}(\boldsymbol{k})= & v_{1}\left[\tau^{x} \cos \left(\frac{k_{x}}{2}\right) \cos \left(\frac{k_{y}}{2}\right)+\tau^{x} \mu^{x} \cos \left(\frac{k_{y}}{2}\right) \cos \left(\frac{k_{z}}{2}\right)\right. \\
& \left.+\mu^{x} \cos \left(\frac{k_{z}}{2}\right) \cos \left(\frac{k_{x}}{2}\right)\right]+v_{p}\left[\tau^{y} \mu^{z} \cos \left(\frac{k_{x}}{2}\right)\right. \\
& \times \sin \left(\frac{k_{y}}{2}\right)+\tau^{y} \mu^{x} \cos \left(\frac{k_{y}}{2}\right) \sin \left(\frac{k_{z}}{2}\right) \\
& \left.+\mu^{y} \cos \left(\frac{k_{z}}{2}\right) \sin \left(\frac{k_{x}}{2}\right)\right], \\
V_{r 1}(\boldsymbol{k})= & v_{r 1}\left[\tau^{y} \mu^{z} \sigma^{y} \cos \left(\frac{k_{x}}{2}\right) \cos \left(\frac{k_{y}}{2}\right)+\tau^{y} \mu^{x} \sigma^{z} \cos \left(\frac{k_{y}}{2}\right)\right. \\
& \left.\times \cos \left(\frac{k_{z}}{2}\right)+\mu^{y} \sigma^{x} \cos \left(\frac{k_{z}}{2}\right) \cos \left(\frac{k_{x}}{2}\right)\right], \\
V_{r 2}(\boldsymbol{k})= & v_{r 2}\left[\tau^{y} \sigma^{z} \cos \left(\frac{k_{x}}{2}\right) \cos \left(\frac{k_{y}}{2}\right)+\tau^{x} \mu^{y} \sigma^{x} \cos \left(\frac{k_{y}}{2}\right)\right. \\
& \left.\times \cos \left(\frac{k_{z}}{2}\right)+\tau^{z} \mu^{y} \sigma^{y} \cos \left(\frac{k_{z}}{2}\right) \cos \left(\frac{k_{x}}{2}\right)\right], \quad(14)
\end{aligned}
$$




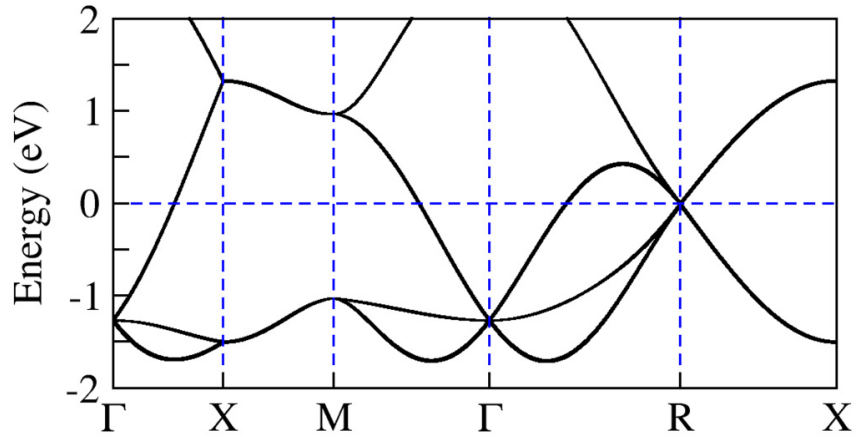

FIG. 2. Energy dispersion of the tight binding model Hamiltonian along high symmetry lines.

$$
\begin{aligned}
V_{r 3}(\boldsymbol{k})= & v_{r 3}\left[\tau^{y} \mu^{z} \sigma^{x} \sin \left(\frac{k_{x}}{2}\right) \sin \left(\frac{k_{y}}{2}\right)+\tau^{y} \mu^{x} \sigma^{y} \sin \left(\frac{k_{y}}{2}\right)\right. \\
& \left.\times \sin \left(\frac{k_{z}}{2}\right)+\mu^{y} \sigma^{z} \sin \left(\frac{k_{z}}{2}\right) \sin \left(\frac{k_{x}}{2}\right)\right], \\
V_{s 1}(\boldsymbol{k})= & v_{s 1}\left[\tau^{x} \sigma^{x} \sin \left(\frac{k_{x}}{2}\right) \cos \left(\frac{k_{y}}{2}\right)+\tau^{x} \mu^{x} \sigma^{y} \sin \left(\frac{k_{y}}{2}\right)\right. \\
& \left.\times \cos \left(\frac{k_{z}}{2}\right)+\mu^{x} \sigma^{z} \sin \left(\frac{k_{z}}{2}\right) \cos \left(\frac{k_{x}}{2}\right)\right], \\
& \left.\times \sin \left(\frac{k_{z}}{2}\right)+\mu^{x} \sigma^{x} \cos \left(\frac{k_{z}}{2}\right) \sin \left(\frac{k_{x}}{2}\right)\right], \\
V_{s 2}(\boldsymbol{k})= & v_{s 2}\left[\tau^{x} \sigma^{y} \cos \left(\frac{k_{x}}{2}\right) \sin \left(\frac{k_{y}}{2}\right)+\tau^{x} \mu^{x} \sigma^{z} \cos \left(\frac{k_{y}}{2}\right)\right. \\
V_{s 3}(\boldsymbol{k})= & v_{s 3}\left[\tau^{x} \mu^{z} \sigma^{z} \cos \left(\frac{k_{x}}{2}\right) \sin \left(\frac{k_{y}}{2}\right)-\tau^{y} \mu^{y} \sigma^{x} \cos \left(\frac{k_{y}}{2}\right)\right. \\
& \left.\times \sin \left(\frac{k_{z}}{2}\right)+\tau^{z} \mu^{x} \sigma^{y} \cos \left(\frac{k_{z}}{2}\right) \sin \left(\frac{k_{x}}{2}\right)\right] .
\end{aligned}
$$

The corresponding energy dispersion along high symmetry directions have been illustrated in Fig. 2.

We first check the Berry curvature distribution in $k$ space. Considering the $\Omega_{z}$ component as an example, the local distribution of $\Omega_{z}$ was computed based on the aforementioned tight-binding model by following

$$
\Omega_{z}=2 \operatorname{Im} \sum_{n<n_{o c c}} \frac{\left\langle n(\boldsymbol{k})\left|\hat{v}_{x}\right| m(\boldsymbol{k})\right\rangle\left\langle m(\boldsymbol{k})\left|\hat{v}_{y}\right| n(\boldsymbol{k})\right\rangle}{\left(E_{\boldsymbol{k}, n}-E_{\boldsymbol{k}, m}\right)^{2}} .
$$

The calculated results have been depicted in Fig. 2(a).

As discussed previously, the chirality of the chiral fermions change the sign of corresponding crystal structures with opposite chirality. Accordingly, since chiral fermions are Berry curvature monopoles, the Berry curvature at any $k$ point around $R$ changes sign after inversion operation.

Similarly, the local distribution of $\tilde{\chi}_{y z}^{C, x}(\boldsymbol{k} ; 0, \omega,-\omega)$ and $\tilde{\chi}_{y z}^{L, x}(\boldsymbol{k} ; 0, \omega,-\omega)$ are computed based on the tight- binding model. The chirality of the Berry curvature is also related to the correlation between the band structure and polarized light. As depicted in Figs. 3(b) and 3(c), when the Fermi level is equal to the linear crossing point, two transitions exist for a selected transition energy on the $k_{z}=0$ plane.

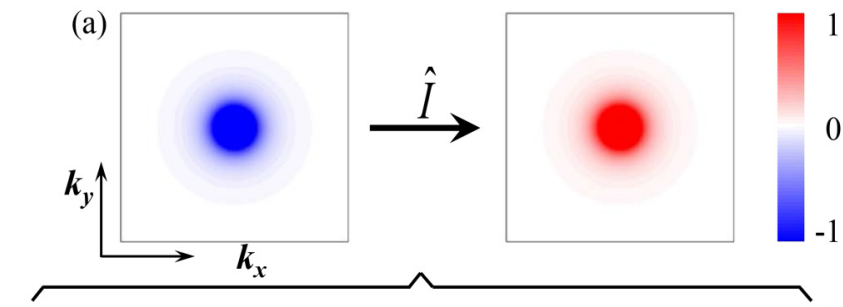

(b)

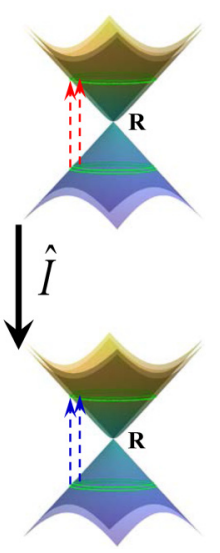

(c)
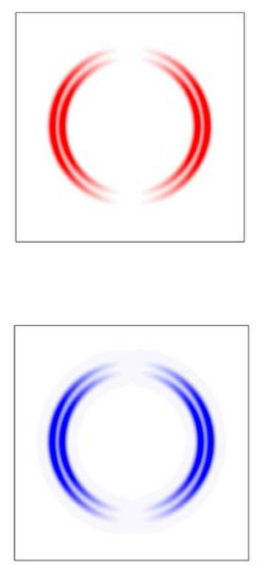

(d)
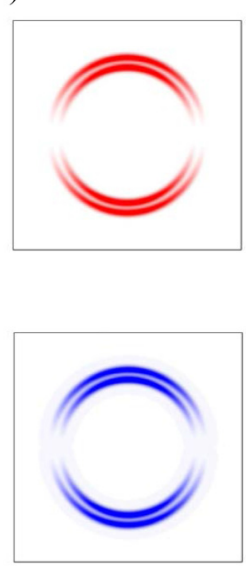

FIG. 3. Local distribution of $\Omega_{z}, \tilde{\chi}_{y z}^{C P G E, x}(\boldsymbol{k} ; 0, \omega,-\omega)$, and $\tilde{\chi}_{y z}^{L P G E, x}(\boldsymbol{k} ; 0, \omega,-\omega)$ around $R$ points from numerical calculations based on a tight binding model of RhSi. (a) Inversion-operationinduced sign change for the local Berry curvature $\left(\Omega_{z}\right)$ distribution around multifold chiral fermions. (b) Light excitation between the lower and upper cones of multifold fermions. The green rings represent the transition path corresponding to a given frequency. The light excitation from the hot ringlike distribution for (c) $\tilde{\chi}_{y z}^{C P G E, x}(\boldsymbol{k} ; 0, \omega,-\omega)$ and (d) $\tilde{\chi}_{y z}^{L P G E, x}(\boldsymbol{k} ; 0, \omega,-\omega) . \tilde{\chi}_{y z}^{C P G E, x}$ and $\tilde{\chi}_{y z}^{L P G E, x}$ change the sign via the inversion operation on the crystal structure. The plot lies on the $k_{z}=\pi$ plane around the point, $R$. The color bars are expressed in arbitrary units. The left panel of (a) and the upper panels of (b)-(d) correspond to the crystal structure depicted in Fig. 1(a). The right panel of (a) and lower panels of (b)-(d) correspond to the crystal structure depicted in Fig. 1(b).

These two transitions form two hot rings for both the distribution functions, $\tilde{\chi}_{y z}^{C, x}(\boldsymbol{k} ; 0, \omega,-\omega)$ and $\tilde{\chi}_{y z}^{L, x}(\boldsymbol{k} ; 0, \omega,-\omega)$, as depicted in the upper panels of Figs. 3(c) and 3(d). The positions of the hot rings remain constant after inversion, but their signs are switched, as illustrated in the bottom panels of Figs. 3(c) and 3(d).

\section{PROPOSED EXPERIMENTAL SETUP FOR DETECTING SIGN CHANGE OF PHOTOCURRENTS}

The second-order conductivity tensors calculated by integrating the local distribution over the entire BZ are completely consistent with the symmetry analysis. Owing to the large separation between opposite-chirality fermions in the energy space, the trace of CPGE is a quantized value in units $i \pi \frac{e^{3}}{h^{2}}$. In Fig. 4(a), the transition-energy-dependent CPGE for $\chi_{y z}^{C, x}$ has been depicted, corresponding to $\beta_{x x}$ in Ref. [8]. Since we consider only one component of the trace, a third of the quantized value exists in the range $\sim 0.1$ to $\sim 0.8 \mathrm{eV}$. On inverting the crystal structure to its counterpart with opposite 
(a)

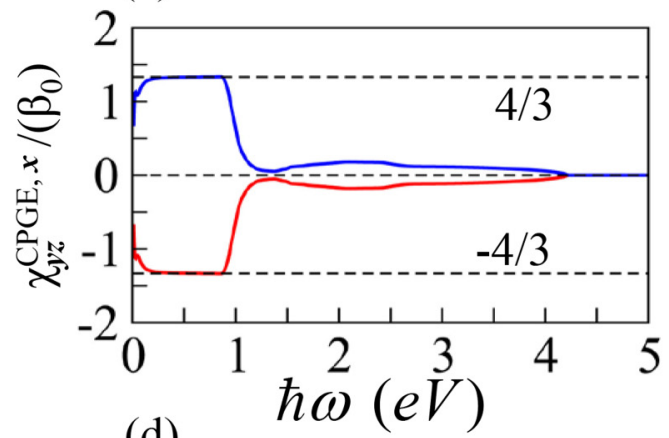

(d)

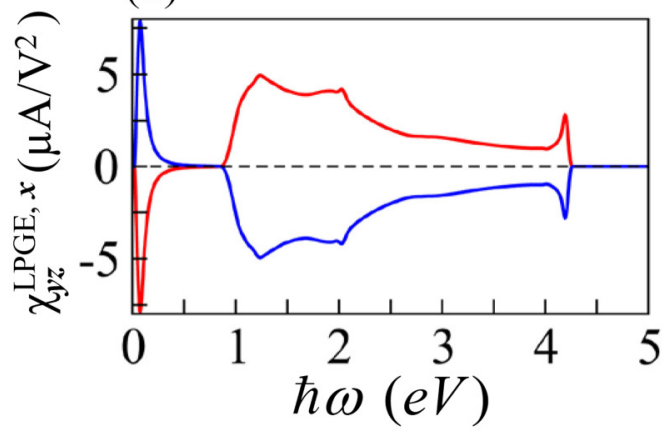

(b)

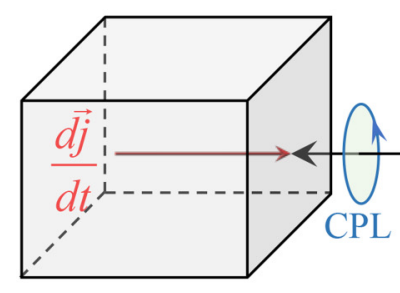

(e)

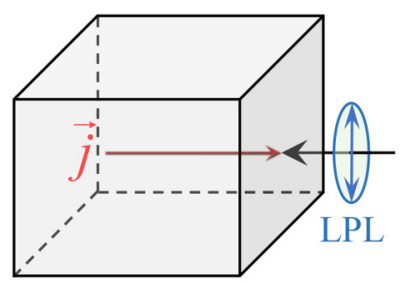

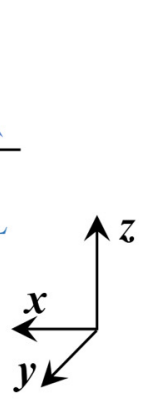

(c)

(f)

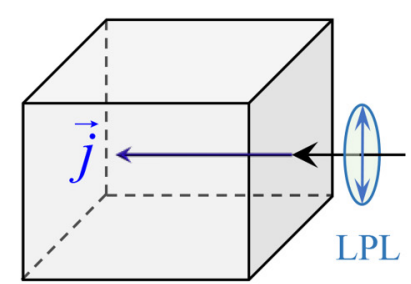

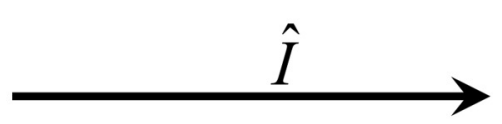

FIG. 4. Frequency-dependent second-order conductivity for (a) CPGE and (d) LPGE based on numerical calculations based on the tightbinding model of RhSi. The red and blue curves correspond to opposite chiralities of the chiral fermions. The signs of all three conductivities change with the switch in chirality of the crystal structure. Schematic of the experimental setup to identify multifold fermions in crystals with opposite chiralities by (b), (c) CPGE and (e), (f) LPGE. CPL in (b), (c) represents circularly polarized light, and LPL in (e), (f) represents linearly polarized light. The calculations were performed based on the tight-binding model reported in Ref. [3] with the parameters $v_{1}=1.0$, $v_{p}=-1.0, v_{r 1}=0.0, v_{r 2}=-0.01, v_{r 3}=0.01, v_{s 1}=-0.01, v_{s 2}=0.0$, and $v_{s 3}=0$ and an on-site term $v_{0}=0.01$ to translate the degeneracy at the point, $R$, to the Fermi level. $\beta_{0}=i \pi \frac{e^{3}}{h^{2}}$ in (a). (b) and (e) correspond to the crystal structure depicted in Fig. 1(a). (c) and (f) correspond to the crystal structure depicted in Fig. 1(b).

chirality, the sign of CPGE changes. Therefore, for circularly polarized light with a specific helix, the generated voltage drop is opposite for the same type of chiral fermions with leftand right-handed chirality.

Based on symmetry and numerical analyses, we present a schematic setup for the experimental measurement of $\chi_{y z}^{C, x}$, as an example. The incidence of circularly polarized light along the $x$ direction, with its electrical field locked on the $y-z$ plane, induces an injection current along $-x$. When the same circularly polarized light is transmitted along the same direction in the same compound with opposite chirality, the sign of the injection current changes, as depicted in Figs. 4(b) and 4(c). Though the LPGE is not quantized, a strong shift current is expected in this class of chiral crystals because of the existence of strong inversion-symmetry breaking [38]. The incidence of linearly polarized light along the same direction induces a shift current along $x$, and the sign corresponding to its counterpart with opposite chirality is changed, as depicted in Figs. 4(d)-4(f). The sign change of the strong signal of the shift current can be easily detected.

\section{SECOND-HARMONIC GENERATION}

In addition to the second order DC photocurrent, the polarized light can also induce a second-harmonic dipole, $P_{c}(2 \omega)=\epsilon_{0} \chi_{a b}^{S H G, c}(\omega) E^{a}(\omega) E^{b}(\omega)$, where $\epsilon_{0}$ denotes the vacuum permittivity, and $\chi_{a b}^{S H G, c}(\omega)$ denotes the second-order susceptibility, which can be computed from two terms of $\chi_{a b}^{S H G, c}(\omega)=\chi_{a b, e}^{S H G, c}(\omega)+\chi_{a b, i}^{S H G, c}(\omega)$. These two terms can be expressed as [39]

$$
\begin{aligned}
\chi_{a b, e}^{S H G, c}(\omega)= & \frac{e^{3}}{2 \hbar^{2}} \sum_{k} \sum_{n m l} \frac{r_{n m}^{a}\left(r_{m l}^{b} r_{l n}^{c}+r_{m l}^{c} r_{l n}^{b}\right)}{\omega_{l n}-\omega_{m l}} \\
& \times\left(\frac{2 f_{n m}}{\omega_{n m}-2 \omega}+\frac{f_{l n}}{\omega_{l n}-\omega}+\frac{f_{m l}}{\omega_{m l}-\omega}\right)
\end{aligned}
$$

and

$$
\begin{aligned}
\chi_{a b, i}^{S H G, c}(\omega)= & \frac{i e^{3}}{2 \hbar^{2}} \sum_{k} \sum_{n m} f_{n m}\left(\frac{2 r_{n m}^{a}\left(r_{m n}^{b ; c}+r_{m n}^{c ; b}\right)}{\omega_{m n}\left(\omega_{m n}-2 \omega\right)}\right. \\
& +\frac{r_{n m}^{a ; c} r_{m n}^{b}+r_{n m}^{a ; b} r_{m n}^{c}}{\omega_{m n}\left(\omega_{m n}-\omega\right)}+\frac{r_{n m}^{a}\left(r_{m n}^{b} \Delta_{m n}^{c}+r_{m n}^{c} \Delta_{m n}^{b}\right)}{\omega_{m n}^{2}} \\
& \times\left(\frac{1}{\omega_{m n}-\omega}-\frac{4}{\omega_{m n}-2 \omega}\right) \\
& \left.-\frac{r_{n m}^{b ; a} r_{m n}^{c}+r_{n m}^{c ; a} r_{m n}^{b}}{2 \omega_{m n}\left(\omega_{m n}-\omega\right)}\right) .
\end{aligned}
$$

The calculated SHGs are depicted in Fig. 5, from which one can also observe the sign change for both the real and imaginary parts of the second-order susceptibility when the 

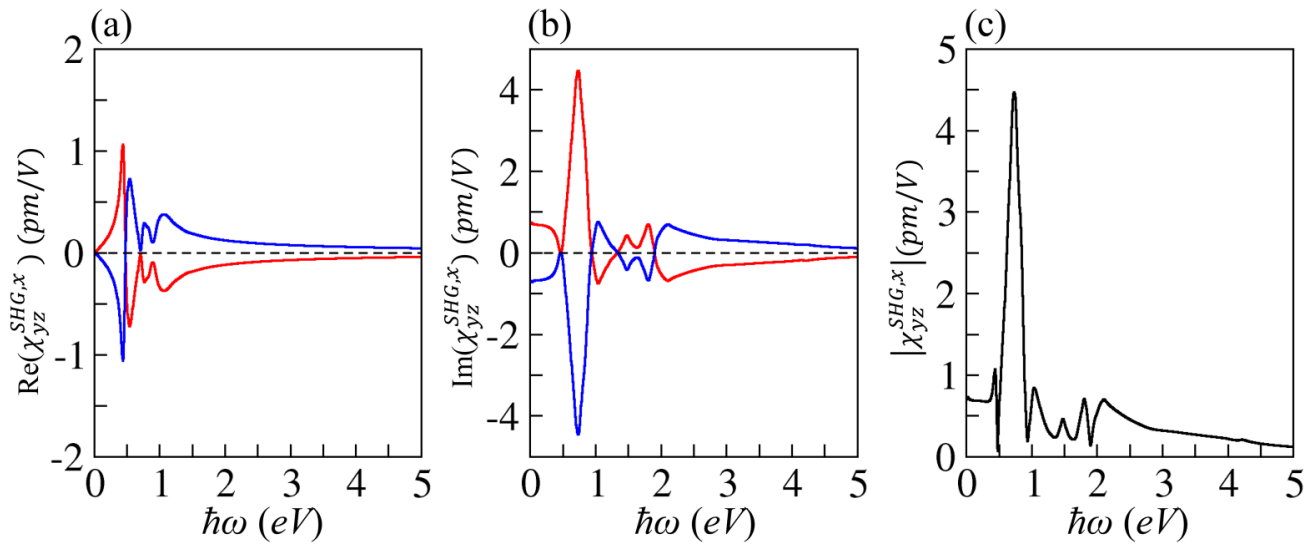

FIG. 5. Frequency dependent second-order susceptibility $\chi_{y z}^{S H G, x}$ for the (a) real and (b) imaginary part, respectively. The red and blue curves correspond to the crystal structures depicted in Fig. 1(a) and Fig. 1(b), respectively. (c) The magnitude of $\chi_{y z}^{S H G, x}$.

crystal structure is inverted. Since the experimental measurements for SHG primarily focus on the magnitude [consult Fig. 5(c)], the modality of the sign change of SHG for the separated real and imaginary parts is relatively complicated compared to the measurement of DC photocurrent.

\section{SUMMARY}

In summary, the switch in chirality of chiral multifold fermions is related to the sign change of charge currents generated by second-order electrical and optical responses. This relationship provides an effective bulk-transport approach to experimentally identify the chirality of chiral fermions of the same type, as well as the relationship between the chirality of chiral fermions in reciprocal space and crystal structures in real space.

\section{ACKNOWLEDGMENTS}

Thanks to I. Sodemann and L. Wu for the helpful discussion. This work was financially supported by the ERC Advanced Grant No. 291472 Idea Heusler, ERC Advanced Grant No. 742068 TOPMAT. This work was performed in part at the Center for Nanoscale Systems (CNS), a member of the National Nanotechnology Coordinated Infrastructure Network (NNCI), which is supported by the National Science Foundation under NSF Award No. 1541959. CNS is part of Harvard University. Some of our calculations were carried out on the Cobra cluster of MPCDF, Max Planck society.
[1] J. L. Manes, Phys. Rev. B 85, 155118 (2012).

[2] B. Bradlyn, J. Cano, Z. Wang, M. Vergniory, C. Felser, R. Cava, and B. A. Bernevig, Science 353, aaf5037 (2016).

[3] G. Chang, S.-Y. Xu, B. J. Wieder, D. S. Sanchez, S.-M. Huang, I. Belopolski, T.-R. Chang, S. Zhang, A. Bansil, H. Lin et al., Phy. Rev. Lett. 119, 206401 (2017).

[4] P. Tang, Q. Zhou, and S.-C. Zhang, Phy. Rev. Lett. 119, 206402 (2017).

[5] G. Chang, B. J. Wieder, F. Schindler, D. S. Sanchez, I. Belopolski, S.-M. Huang, B. Singh, D. Wu, T.-R. Chang, T. Neupert, et al., Nat. Mater. 17, 978 (2018).

[6] T. Zhang, Z. Song, A. Alexandradinata, H. Weng, C. Fang, L. Lu, and Z. Fang, Phys. Rev. Lett. 120, 016401 (2018).

[7] F. Flicker, F. de Juan, B. Bradlyn, T. Morimoto, M. G. Vergniory, and A. G. Grushin, Phys. Rev. B 98, 155145 (2018).

[8] F. de Juan, A. G. Grushin, T. Morimoto, and J. E. Moore, Nat. Commun. 8, 15995 (2017).

[9] D. S. Sanchez, I. Belopolski, T. A. Cochran, X. Xu, J.-X. Yin, G. Chang, W. Xie, K. Manna, V. Süß, C.-Y. Huang, N. Alidoust, D. Multer, S. S. Zhang, N. Shumiya, X. Wang, G.-Q. Wang, t.-r. Chang, C. Felser, S.-Y. Xu, S. Jia, H. Lin, and M. Z. Hasan, Nature (London) 567, 500 (2019).
[10] N. B. Schröter, D. Pei, M. G. Vergniory, Y. Sun, K. Manna, F. de Juan, J. A. Krieger, V. Süss, M. Schmidt, P. Dudin, B. Bradlyn, T. K. Kim, T. Schmitt, C. Cacho, C. Felser, V. N. Strocov, and Y. Chen, Nat. Phys. 15, 759 (2019).

[11] Z. Rao, H. Li, T. Zhang, S. Tian, C. Li, B. Fu, C. Tang, L. Wang, Z. Li, W. Fan, J. Li, Y. Huang, Z. Liu, Y. Long, C. Fang, H. Weng, Y. Shi, H. Lei, Y. Sun, T. Qian, and H. Ding, Nature (London) 567, 496 (2019).

[12] D. Takane, Z. Wang, S. Souma, K. Nakayama, T. Nakamura, H. Oinuma, Y. Nakata, H. Iwasawa, C. Cacho, T. Kim, K. Horiba, H. Kumigashira, T. Takahashi, Y. Ando, and T. Sato, Phys. Rev. Lett. 122, 076402 (2019).

[13] N. Schröter, S. Stolz, K. Manna, F. de Juan, M. G. Vergniory, J. A. Krieger, D. Pei, P. Dudin, T. K. Kim, C. Cacho et al., Science 369, 179 (2020)

[14] P. Sessi, F.-R. Fan, F. Küster, K. Manna, N. Schröter, J.-R. Ji, S. Stolz, J. A. Krieger, D. Pei, T. K. Kim et al., Nat. Commun. 11, 3507 (2020).

[15] D. Rees, K. Manna, B. Lu, T. Morimoto, H. Borrmann, C. Felser, J. Moore, D. H. Torchinsky, and J. Orenstein, Sci. Adv. 6, eaba0509 (2020).

[16] B. Xu, Z. Fang, M. A. Sánchez-Martínez, J. W. F. Venderbos, Z. Ni, T. Qiu, K. Manna, K. Wang, J. Paglione, C. Bernhard et al., arXiv:2005.01581 (2020). 
[17] Z. Ni, B. Xu, M. Sanchez-Martinez, Y. Zhang, K. Manna, C. Bernhard, J. W. F. Venderbos, F. de Juan, C. Felser, A. G. Grushin, and L. Wu, arXiv:2005.13473 (2020).

[18] H. Li, X. Sheng, L.-Q. Rao, Zhi-Cheng Zhou, Z.-J. Wang, S.J. T. Tian, S.-Y. Gao, J.-J. Li, Y.-B. Huang, H.-C. Lei, Y.-J. Sun, T.-L. Xia, T. Qian, and H. Ding, Nat. Commun. 10, 5505 (2019).

[19] Y. Tokura, M. Kawasaki, and N. Nagaosa, Nat. Phys. 13, 1056 (2017).

[20] G. Lefkidis, G. P. Zhang, and W. Hübner, Phys. Rev. Lett. 103, 217401 (2009).

[21] J.-P. Hanke, F. Freimuth, A. K. Nandy, H. Zhang, S. Blügel, and Y. Mokrousov, Phys. Rev. B 94, 121114(R) (2016).

[22] M. Fiebig, D. Fröhlich, Th. Lottermoser, V. V. Pavlov, R. V. Pisarev, and H.-J. Weber, Phys. Rev. Lett. 87, 137202 (2001).

[23] Y. Zhang, T. Holder, H. Ishizuka, F. de Juan, N. Nagaosa, C. Felser, and B. Yan, Nat. Commun. 10, 1 (2019).

[24] W. Kraut and R. von Baltz, Phys. Rev. B 19, 1548 (1979).

[25] V. Belinicher and B. Sturman, Phys.- Usp. 23, 199 (1980).

[26] J. E. Sipe and A. I. Shkrebtii, Phys. Rev. B 61, 5337 (2000).

[27] I. Sodemann and L. Fu, Phys. Rev. Lett. 115, 216806 (2015).

[28] Q. Ma, S.-Y. Xu, H. Shen, D. MacNeill, V. Fatemi, T.-R. Chang, A. M. M. Valdivia, S. Wu, Z. Du, C.-H. Hsu, et al., Nature (London) 565, 337 (2019).
[29] N. Kristoffel and A. Gulbis, Z. Phys. B Condensed Matter 39, 143 (1980).

[30] R. von Baltz and W. Kraut, Phys. Rev. B 23, 5590 (1981).

[31] N. Kristoffel, R. Von Baltz, and D. Hornung, Z. Phys. B Condensed Matter 47, 293 (1982).

[32] H. Presting and R. Von Baltz, Phys. Status Solidi B 112, 559 (1982).

[33] C. Aversa and J. E. Sipe, Phys. Rev. B 52, 14636 (1995).

[34] K. Yao, B. K. Gan, M. Chen, and S. Shannigrahi, Appl. Phys. Lett. 87, 212906 (2005).

[35] Q. Ma, S.-Y. Xu, C.-K. Chan, C.-L. Zhang, G. Chang, Y. Lin, W. Xie, T. Palacios, H. Lin, S. Jia et al., Nat. Phys. 13, 842 (2017).

[36] S.-Y. Xu, Q. Ma, H. Shen, V. Fatemi, S. Wu, T.-R. Chang, G. Chang, A. M. M. Valdivia, C.-K. Chan, Q. D. Gibson, et al., Nat. Phys. 14, 900 (2018).

[37] T. Morimoto, M. Nakamura, M. Kawasaki, and N. Nagaosa, Phys. Rev. Lett. 121, 267401 (2018).

[38] Y. Zhang, F. de Juan, A. G. Grushin, C. Felser, and Y. Sun, Phys. Rev. B 100, 245206 (2019).

[39] S. N. Rashkeev, W. R. L. Lambrecht, and B. Segall, Phys. Rev. B 57, 3905 (1998). 